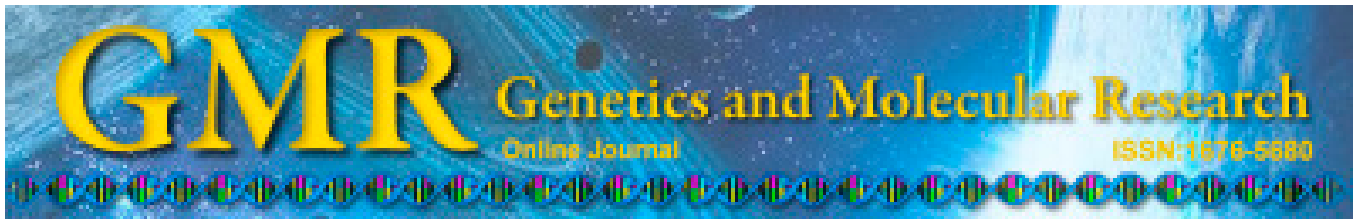

\title{
Meta-analysis of angiotensin-converting enzyme insertion/deletion polymorphism and myocardial infarction in Han Chinese
}

\author{
W. Zhao, ${ }^{1,2}$, S.T. Ma ${ }^{1,3}$ and L.Q. Cui ${ }^{1}$ \\ ${ }^{1}$ Department of Cardiology, \\ The Provincial Hospital Affiliated to Shandong University, \\ Shandong Province, China \\ ${ }^{2}$ Department of Cardiology, Shandong Jiaotong Hospital, \\ Shandong Province, China \\ ${ }^{3}$ Department of Cardiology, The Fourth Hospital of Jinan, \\ Shandong Province, China
}

Corresponding author: L.Q. Cui

E-mail: cuilqmed@126.com

Genet. Mol. Res. 14 (3): 8068-8076 (2015)

Received November 13, 2014

Accepted April 7, 2015

Published July 17, 2015

DOI http://dx.doi.org/10.4238/2015.July.17.15

\begin{abstract}
It has been suggested that the angiotensin-converting enzyme (ACE) gene insertion/deletion (I/D) polymorphism is linked to susceptibility to myocardial infarction (MI). In this study, we performed a meta-analysis to assess the relationship between ACE I/D polymorphism and MI in the Chinese Han population. Eight studies including a total of 1609 subjects were selected for inclusion in the analysis. The references were retrieved using the PubMed and China National Knowledge Infrastructure databases. The analyses were performed using the STATA 12.0 software. ORs and 95\%CI were assessed after the collected data were pooled for analysis. There was a significant association between ACE I/D polymorphism and MI in the Chinese Han population (II vs DD: OR $=0.40,95 \% \mathrm{CI}=0.31$ -
\end{abstract}


0.53 ; II $v s$ DI: $\mathrm{OR}=0.72,95 \% \mathrm{CI}=0.57-0.91$; the dominant model: $\mathrm{OR}=1.74,95 \% \mathrm{CI}=1.41-2.16$; the recessive model: $\mathrm{OR}=0.47$, $95 \% \mathrm{CI}=0.38-0.60)$. The sensitivity analysis further confirmed the result. Publication bias was not observed in this meta-analysis. The ACE I/D polymorphism may be a risk factor for MI in the Chinese Han population. However, larger studies with a stratified case-control population and biological characterization are needed to validate this finding.

Key words: Angiotensin-converting enzyme; Meta-analysis; Insertion/deletion polymorphism; Myocardial infarction

\section{INTRODUCTION}

Myocardial infarction (MI) remains one of the major causes of death and disability worldwide, accounting for up to $40 \%$ of all deaths (White and Chew, 2008). According to data from the National Health and Nutrition Examination Survey (2003 to 2006) the overall prevalence of MI is 3.6\% in US adults over the age of 20, with rates of $4.7 \%$ for men and $2.6 \%$ for women (Schiller et al., 2012). Despite much investigation, the causes are not yet fully understood. The classic risk factors for MI include hypertension, hypercholesterolemia, diabetes, obesity, and smoking. In addition, genetic factors play an important role in the development of the disorder. It has been estimated that approximately $50 \%$ of the major risk factors for coronary artery disease are determined by genetics (Sekuri et al., 2005).

The renin-angiotensin-aldosterone system (RAAS) is essential to cardiovascular hemodynamics and plays an important role in the development of hypertension and cerebrovascular diseases. Angiotensin-converting enzyme (ACE) is an important circulating enzyme in the RAAS. It catalyzes the conversion of angiotensin I to angiotensin II and degrades bradykinin. The human ACE gene has the chromosomal locus 17q23 and many polymorphisms have been identified. The polymorphism rs4646994 is characterized by the presence or absence of a 287-bp Alu repetitive sequence, which results in three genotypes: II, DI, and DD (Haiman et al., 2003). The DD genotype is associated with two-fold higher tissue and plasma concentrations of ACE than the II genotype (Rigat et al., 1990). The ACE insertion/deletion (I/D) polymorphism has therefore been extensively studied in several cardiovascular and cerebrovascular diseases, such as ischemic stroke and coronary artery disease (Gao et al., 2006; Vaisi-Raygani et al., 2010).

In the past decade, a number of epidemiological studies have assessed the association between the ACE I/D polymorphism and MI risk. However, the reported results are controversial. A recent meta-analysis indicated that the ACE I/D polymorphism is related to an increased risk of MI (Chen et al., 2013). However, that meta-analysis included only limited articles with Chinese ancestry; most of the studies performed on Chinese participants were published in Chinese and in Chinese journals. Therefore, it is very important to clarify the relationship between ACE I/D polymorphism and MI risk in Chinese populations. In the present study, we investigated the association between ACE I/D polymorphism and MI risk in a Chinese population by performing a meta-analysis of the data from the literature. 


\section{MATERIAL AND METHODS}

\section{Selection of studies}

Two reviewers searched PubMed and China National Knowledge Infrastructure (CNKI) databases to retrieve papers linking ACE I/D polymorphism and MI risk, which were available from October 2014 and had no language restrictions, using the following key words: “ACE I/D polymorphism", "myocardial infarction/MI", "rs4646994", "single nucleotide polymorphism", and "genetic polymorphism". The reference lists of major textbooks, reviews, and included articles were identified through manual searches to find other potentially eligible studies. When more than one study of the same population was identified, we included the study with the largest sample size. When pertinent data were not included, or data presented were unclear, the authors were contacted directly.

\section{Study selection}

To be eligible for inclusion in this meta-analysis, the following criteria were stipulated: i) case-control studies must have addressed MI cases and healthy controls; ii) studies must have been about the association between ACE I/D polymorphism and susceptibility to MI; and iii) studies must have included sufficient genotype data for extraction. The exclusion criteria were as follows: i) case-control studies that did not evaluate the association between ACE I/D polymorphism and MI risk; ii) case reports, letters, reviews, meta-analyses, and editorial articles; iii) studies that were based on incomplete raw data and those with no usable data reported; and iv) duplicate data.

\section{Data extraction}

Two investigators independently extracted data and reached a consensus on all the items. The following information was recorded for each study: first author, year of publication, country, nationality, number of patients and controls, polymorphisms of gene, and evidence of Hardy-Weinberg equilibrium (HWE). In the case of conflicting evaluations, an agreement was reached following a discussion.

\section{Statistical analysis}

Observed genotype frequencies for ACE I/D polymorphism in controls were examined for deviations from HWE using a goodness-of-fit $\chi^{2}$-test with one degree of freedom. The strength of the associations between ACE I/D polymorphism and susceptibility to MI was estimated by OR and 95\%CI of a homozygote comparison (II vs DD), a heterozygote comparison (II vs DI), a dominant model (DD + DI $v s$ II), and a recessive model (II + DI $v s$ DD) between groups (Lin et al., 2011). The statistical heterogeneity among studies was assessed using $\mathrm{I}^{2}$ statistics (Higgins et al., 2003). $\mathrm{I}^{2}$ ranges between 0 and $100 \%$ and represents the proportion of inter-study variability that can be attributed to heterogeneity rather than chance. $\mathrm{I}^{2}$ values of 25,50 , and $75 \%$ were defined as low, moderate, and high estimates, respectively. When $\mathrm{I}^{2}>50 \%$ indicated heterogeneity across studies, the random-effects model 
was used for meta-analysis; otherwise, the fixed-effects model was used. Sensitivity analysis was performed through random-effect model values compared with the fixed effect. We also performed a cumulative meta-analysis to provide a framework for updating the genetic effect from all studies, and to determine how the genetic effect changed as evidence accumulated to ascertain the trend in estimated risk effect (Zintzaras and Lau, 2008). In cumulative metaanalysis, studies were chronologically ordered by publication year, and then the pooled ORs were obtained at the end of each year. The funnel plot asymmetry was assessed by Begg's test to estimate the potential publication bias $(\mathrm{P}<0.05$ was considered to be statistically significant). Meta-analysis was performed using the STATA package version 12.0 (Stata Corporation, College Station, TX, USA).

\section{RESULTS}

\section{Study characteristics}

A total of 58 potentially relevant publications up to October 2014 were systematically identified through PubMed and CNKI databases. Based on our preliminary search criteria, 50 were excluded because they did not satisfy the inclusion criteria. A total of 828 cases and 781 controls were included in the meta-analysis (Lu et al., 1997; Zheng et al., 1997; Lv et al., 1998; Li et al., 1999; Zhao et al., 2002; Sun et al., 2006; Zhang et al., 2006; Wang et al., 2010). The characteristics of the studies selected are summarized in Figure 1. Genotyping was conducted using polymerase chain reaction/restriction fragment length polymorphism for all studies. All studies were case-control studies that evaluated the association between ACE I/D polymorphism and MI risk. The years of publication of the studies included ranged from 1996 to 2010. The HWE test was conducted on the genotype distribution of the controls in all included studies, and all studies met the HWE requirement except three (Li et al., 1999; Zhao et al., 2002; Sun et al., 2006). The baseline characteristics of all studies included are summarized in Table 1.

\section{Results of meta-analyses}

A summary of the meta-analysis findings of the association between ACE I/D polymorphism and MI risk is shown in Table 2 and Figure 2. The meta-analysis indicated that the ACE $\mathrm{I} / \mathrm{D}$ polymorphism was significantly associated with $\mathrm{MI}$ in all the genetic models (II vs DD: OR $=0.40,95 \% \mathrm{CI}=0.31-0.53$; II $v$ S DI: OR $=0.72,95 \% \mathrm{CI}=0.57-0.91$; the dominant model: $\mathrm{OR}$ $=1.74,95 \% \mathrm{CI}=1.41-2.16$; the recessive model: $\mathrm{OR}=0.47,95 \% \mathrm{CI}=0.38-0.60)$. The cumulative meta-analysis showed an increasing trend in the estimated risk effect during 1996 to 2010, which showed that ACE I/D polymorphism is associated with MI risk, and the results were stable (Figure 3). Furthermore, sensitivity analysis was performed with controls in the HWE and the result was not altered, indicating that the result of meta-analysis was statistically significant (II $v s$ DD: $\mathrm{OR}=0.37,95 \% \mathrm{CI}=0.25-0.54$; II $v s \mathrm{DI}: \mathrm{OR}=0.80,95 \% \mathrm{CI}=0.58-1.11$; the dominant model: $\mathrm{OR}=1.65,95 \% \mathrm{CI}=1.23-2.23$; the recessive model: $\mathrm{OR}=0.43,95 \% \mathrm{CI}=0.31-0.60$ ).

\section{Publication bias}

The publication bias of the meta-analysis of the association between ACE I/D poly- 
morphism and MI risk was detected by Begg's funnel plot; all graphical funnel plots of the studies included appeared to be symmetrical (Figure 4). The results of the Begg's funnel plot are shown in Table 2. Results showed that there was no publication bias (all $\mathrm{P}>0.05$ ).

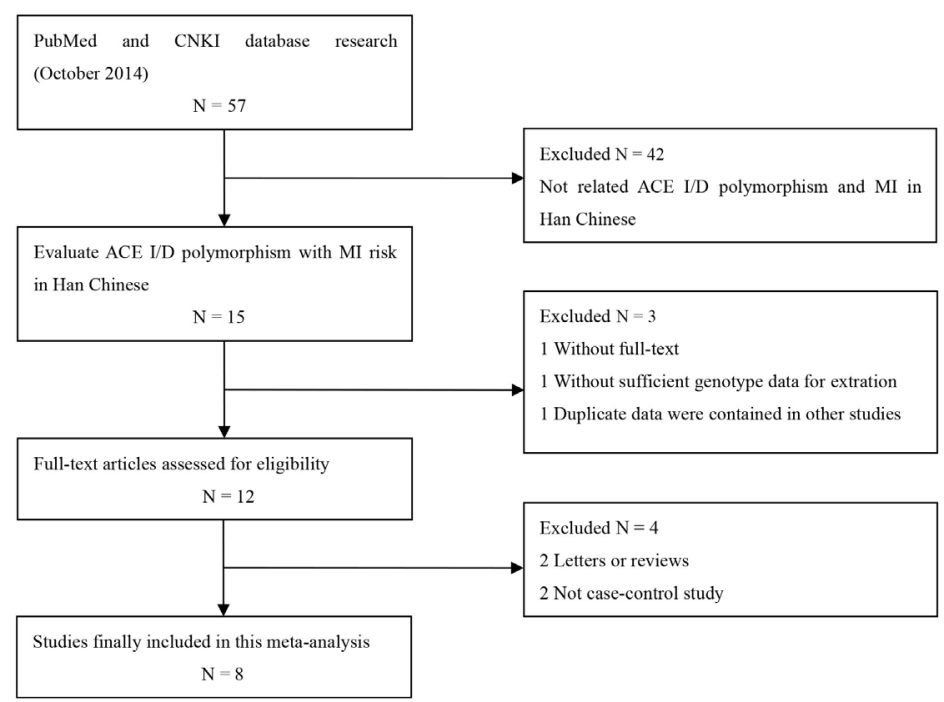

Figure 1. Flow chart showing study selection procedure.

\section{Table 1. Characteristics of the studies included for meta-analysis.}

\begin{tabular}{|c|c|c|c|c|c|c|c|c|c|c|c|}
\hline \multirow[t]{2}{*}{ Study included } & \multirow[t]{2}{*}{ Year } & \multirow[t]{2}{*}{ Area } & \multirow[t]{2}{*}{ Cases } & \multirow[t]{2}{*}{ Controls } & \multicolumn{3}{|c|}{ Genotypes for cases } & \multicolumn{3}{|c|}{ Genotypes for controls } & \multirow[t]{2}{*}{ HWE test } \\
\hline & & & & & DD & ID & II & DD & ID & II & \\
\hline Lu et al. & 1997 & Shanghai & 116 & 103 & 31 & 44 & 41 & 12 & 44 & 47 & 0.73 \\
\hline Zheng et al. & 1997 & Beijing & 103 & 96 & 40 & 46 & 17 & 26 & 40 & 30 & 0.11 \\
\hline Lv et al. & 1998 & Shandong & 58 & 41 & 24 & 18 & 16 & 9 & 17 & 15 & 0.33 \\
\hline Li et al. & 1999 & Beijing & 153 & 150 & 32 & 63 & 58 & 29 & 48 & 73 & 0.00 \\
\hline Zhao et al. & 2002 & Heilongjiang & 140 & 57 & 73 & 40 & 27 & 16 & 19 & 22 & 0.01 \\
\hline Sun et al. & 2006 & Nanjing & 112 & 128 & 36 & 39 & 37 & 20 & 46 & 62 & 0.03 \\
\hline Zhang et al. & 2006 & Guangzhou & 93 & 97 & 30 & 41 & 22 & 11 & 43 & 33 & 0.60 \\
\hline Wang et al. & 2010 & Nanchang & 53 & 109 & 21 & 17 & 15 & 27 & 49 & 33 & 0.31 \\
\hline
\end{tabular}

Table 2. Summary ORs and $95 \% \mathrm{CI}$ of the studies included for meta-analysis.

\begin{tabular}{|c|c|c|c|c|c|c|c|c|c|c|}
\hline \multirow[t]{2}{*}{ Subgroup } & \multirow[t]{2}{*}{ Genetic model } & \multicolumn{2}{|c|}{ Sample size } & \multirow[t]{2}{*}{ Type of model } & \multicolumn{2}{|c|}{ Test of heterogeneity } & \multicolumn{2}{|c|}{ Test of association } & \multicolumn{2}{|c|}{ Test of publication bias } \\
\hline & & Case & Control & & $\mathrm{I}^{2}$ & $P$ & OR & $95 \% \mathrm{CI}$ & $\mathrm{z}$ & $\mathrm{P}$ \\
\hline \multirow[t]{4}{*}{ Overall } & II $v s$ DD & 828 & 781 & Fixed & $1.2 \%$ & 0.42 & 0.40 & $0.31-0.53$ & 0.00 & 1.00 \\
\hline & II $v s \mathrm{DI}$ & & & Fixed & $0.0 \%$ & 0.70 & 0.72 & $0.57-0.91$ & 0.00 & 1.00 \\
\hline & Dominant model & & & Fixed & $0.0 \%$ & 0.73 & 1.74 & $1.41-2.16$ & 0.00 & 1.00 \\
\hline & Recessive model & & & Fixed & $18.7 \%$ & 0.28 & 0.47 & $0.38-0.60$ & 0.00 & 1.00 \\
\hline $\begin{array}{l}\text { Consistent } \\
\text { with }\end{array}$ & II $v s$ DD & 423 & 446 & Fixed & $0.0 \%$ & 0.72 & 0.37 & $0.25-0.54$ & 0.00 & 1.00 \\
\hline \multirow[t]{3}{*}{ HWE } & II $v s$ DI & & & Fixed & $0.0 \%$ & 0.48 & 0.80 & $0.58-1.11$ & 0.00 & 1.00 \\
\hline & Dominant model & & & Fixed & $0.0 \%$ & 0.64 & 1.65 & $1.23-2.23$ & 0.00 & 1.00 \\
\hline & Recessive model & & & Fixed & $0.0 \%$ & 0.70 & 0.43 & $0.31-0.60$ & 0.00 & 1.00 \\
\hline
\end{tabular}




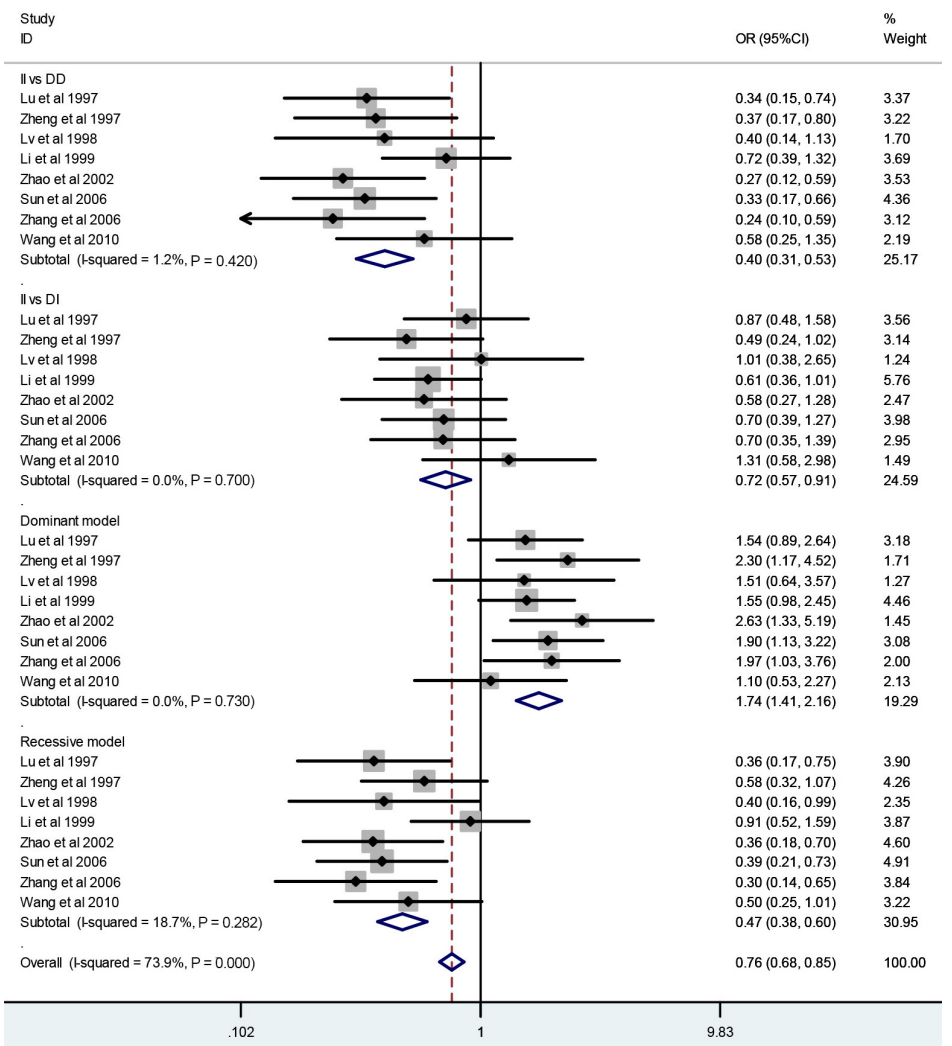

Figure 2. Forest plot of MI risk associated with ACE I/D polymorphism in the Chinese Han population.

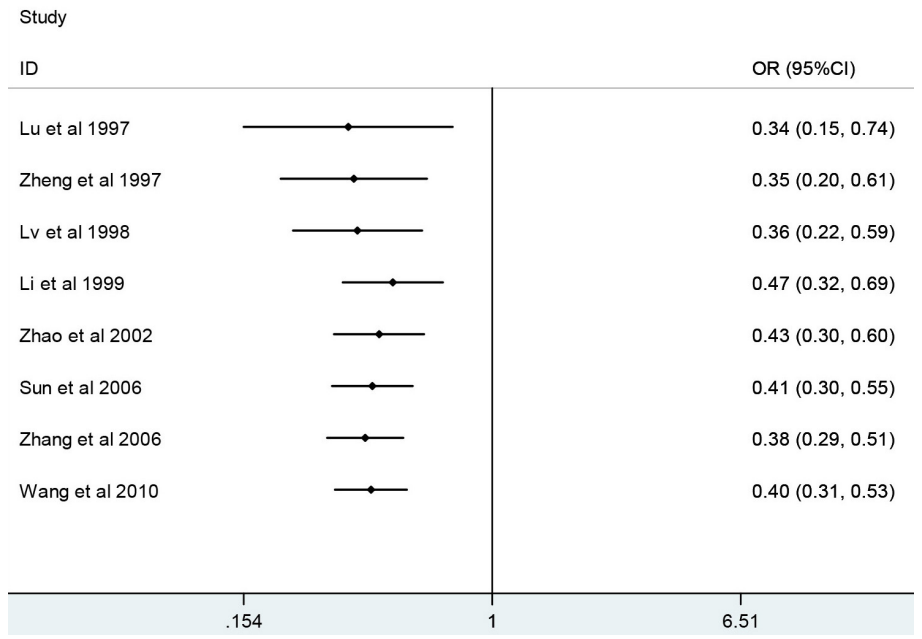

Figure 3. Cumulative meta-analysis for ACE I/D polymorphism in the fixed-effects pooled odds ratios (OR) with the corresponding confidence interval at $95 \%(95 \% \mathrm{CI})$. 


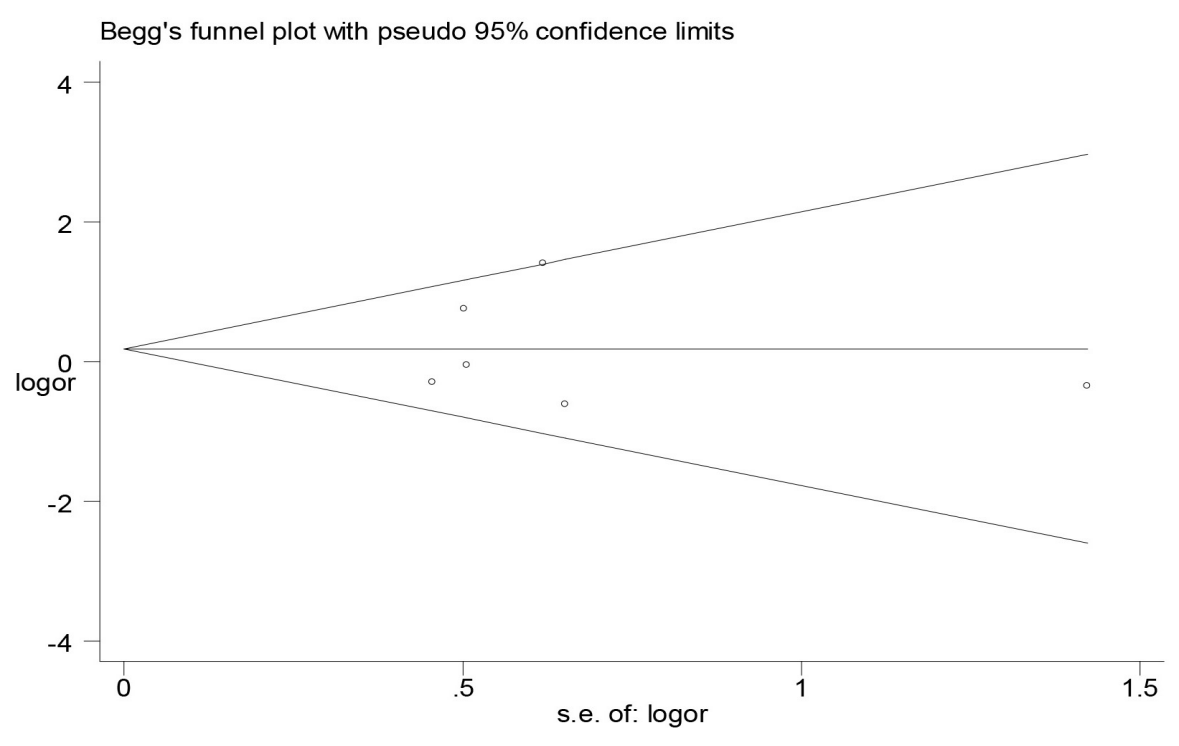

Figure 4. Funnel plot of ACE I/D polymorphism and susceptibility of MI for II vs DD.

\section{DISCUSSION}

MI is a multifactorial disease and its pathogenesis is not yet fully understood. Accumulated evidence indicates incontestably that MI is determined by a complex interaction of environmental and genetic factors. The RAAS plays a vital role in the cardiovascular system, and ACE is one of the most important components of the RAAS. Lu et al. (1997) were the first to report that ACE I/D polymorphism was associated with an increased risk of MI in Han Chinese using a case-control study. However, there has been some inconsistency, with some studies supporting this finding and others contradicting it, which suggests that the observed associations between the ACE I/D polymorphism and increased MI risk reflect chance observations rather than true associations. To help resolve these conflicting results, we conducted this meta-analysis to combine the same kinds of study to increase the sample size and statistical power, and thereby achieve a more authentic result.

In this meta-analysis, we extracted data from eight eligible studies comprising a total of $828 \mathrm{MI}$ patients and 781 controls; the population studied was confined to Chinese subjects with homogeneous genetic backgrounds. Our results suggested that ACE I/D polymorphism was significantly associated with MI in the Chinese Han population. The finding was consistent with those from the previous meta-analysis, which indicated that the association was significant among Europeans and East Asians (Chen et al., 2013). Moreover, the existing evidence of the cumulative meta-analyses is sufficient to suggest that there is no statistically significant association between the ACE I/D polymorphism and MI risk. HWE is essential for a sound case-control study. It is probable that studies without HWE in controls have selection bias or genotyping error, which may cause misleading results. In this meta-analysis, we found that the ACE I/D polymorphism was significantly associated with the risk of MI in studies with $\mathrm{P}_{\mathrm{HWE}}>0.05$. The data of the present meta-analysis indicated that the selection biases 
hardly affected the results. There was no evidence of publication bias in this meta-analysis for ACE I/D polymorphism. As the number of eligible studies was limited in the meta-analysis, caution should be exercised when considering this conclusion.

Some limitations of this meta-analysis should be acknowledged. Firstly, because of incomplete raw data or publication limitations, some relevant studies could not be included in our analysis. Secondly, the small sample size available was not ideal for detecting small genetic effects. Thirdly, the effects of gene-gene and gene-environment interactions were not addressed in this meta-analysis. In addition, the lack of genotype frequency information provided by some published studies did not allow the estimation of the best genetic model of inheritance to follow. Finally, although all cases and controls of each study were well defined with similar inclusion criteria, there may be potential factors that were not taken into account that may have influenced our results.

In conclusion, our results suggest that ACE I/D polymorphism was involved with the susceptibility of MI in the Chinese Han population. Owing to the mentioned limitations, further research should be performed to investigate these associations.

\section{Conflicts of interest}

The authors declare no conflict of interest.

\section{REFERENCES}

Chen Y, Dong S, He M, Qi T, et al. (2013). Angiotensin-converting enzyme insertion/deletion polymorphism and risk of myocardial infarction in an updated meta-analysis based on 34993 participants. Gene 522: 196-205.

Gao X, Yang H and ZhiPing T (2006). Association studies of genetic polymorphism, environmental factors and their interaction in ischemic stroke. Neurosci. Lett. 398: 172-177.

Haiman CA, Henderson SO, Bretsky P, Kolonel LN, et al. (2003). Genetic variation in angiotensin I-converting enzyme (ACE) and breast cancer risk: the multiethnic cohort. Cancer Res. 63: 6984-6987.

Higgins JP, Thompson SG, Deeks JJ and Altman DG (2003). Measuring inconsistency in meta-analyses. BMJ 327: $557-$ 560.

Li Y, Guo JX, Chen DF, Guan BX, et al. (1999). Association study of angiotensin converting enzyme polymorphism with myocardial infarction in Chinese. J. Beijing Med. Univ. 31: 540-542.

Lin G, Fang F, Yu XJ and Yu L (2011). Meta-analysis of the relationship between p21 Ser31Arg polymorphism and lung cancer susceptibility. Genet. Mol. Res. 10: 2449-2456.

Lu L, Yu JD, Kuang SQ, Shen WF, et al. (1997). The relationship between insertion/deletion polymorphism of angiotensinconverting enzyme gene and myocardial infarction in a Chinese population. Chin. J. Cardiol. 25: 30-33.

Lv WZ, Li HJ, Liu SP, Zhou JS, et al. (1998). Polymorphism in angiotensin converting enzyme gene of patients with myocardial infarction and their offsprings. Qilu Med. J. 13: 243-244.

Rigat B, Hubert C, Alhenc-Gelas F, Cambien F, et al. (1990). An insertion/deletion polymorphism in the angiotensin I-converting enzyme gene accounting for half the variance of serum enzyme levels. J. Clin. Invest. 86: 1343-1346.

Schiller JS, Lucas JW, Ward BW and Peregoy JA (2012). Summary health statistics for U.S. adults: National Health Interview Survey, 2010. Vital Health Stat. 10: 1-207.

Sekuri C, Cam FS, Ercan E, Tengiz I, et al. (2005). Renin-angiotensin system gene polymorphisms and premature coronary heart disease. J. Renin Angiotensin Aldosterone Syst. 6: 38-42.

Sun L, Hu WZ, Yang JM, Hong M, et al. (2006). Association of the Angiotensin Converting Enzyme I/D and Angiotensinogen T174M Gene Polymorphism with Acute Myocardial Infarction. Chin. J. Arterioscler. 14: 697-700.

Vaisi-Raygani A, Ghaneialvar H, Rahimi Z, Nomani H, et al. (2010). The angiotensin converting enzyme D allele is an independent risk factor for early onset coronary artery disease. Clin. Biochem. 43: 1189-1194.

Wang SQ, Wu YQ, Cheng XS, Cheng KC, et al. (2010). Relationship between angiotensin-converting enzyme and angiotensinogen gene polymorphism and coronary artery disease. J. Nanchang Med. Univ. 50: 8-11.

White HD and Chew DP (2008). Acute myocardial infarction. Lancet 372: 570-584. 
Zhang YL, Zhou SX, Zhao XY and Lei J (2006). Relationship between polymorphism of angiotensin I converting enzyme gene insertion/deletion and ACE, PAI-I activity in patients with myocardial infarction. Chin. J. Pathophysiol. 22: 2336-2339.

Zhao J, Qu XF and Miao RF (2002). Relationship between angiotensin-1 converting enzyme gene polymorphism and acute myocardial infarction. Chin. J. Crit. Care Med. 22: 444-446.

Zheng HY, Dai YH and Qiu CC (1997). Insertion/deletion polymorphism of ACE gene is associated with serum ACE concentration and increased risk for myocardial infarction in Chinese. Chin. J. Cardiol. 25: 34-36.

Zintzaras E and Lau J (2008). Synthesis of genetic association studies for pertinent gene-disease associations requires appropriate methodological and statistical approaches. J. Clin. Epidemiol. 61: 634-645. 